\title{
Direct left bundle branch pacing can result in dyssynchronous left ventricular contraction and worsening heart failure: a case report
}

\author{
Hrak Chemchirian ${ }^{1}$, Michael Orlov ${ }^{1}$, Michael Maysky ${ }^{1}$, and James Armstrong ${ }^{2}$ \\ ${ }^{1}$ Tufts University School of Medicine \\ ${ }^{2}$ Steward St. Elizabeth's Medical Center
}

June 22, 2020

\begin{abstract}
A 69 year old male patient with mild non ischemic cardiomyopathy, baseline EF of $47 \%$, received a dual chamber pacemaker with a direct left bundle branch lead for complete heart block. 3830 lead was inserted intraseptally according to published recommendations with a resultant QRS of $103 \mathrm{~ms}$. One month later patient presented with severe heart failure. Echocardiogram showed significant dyssynchrony and EF of $21 \%$. Patient remained highly symptomatic despite aggressive medical therapy and exclusion of other causes of heart failure. An upgrade to a bi-ventricular pacing system was performed. At 1 month follow up, patient was no longer exhibiting heart failure symptoms, EF had improved back to baseline (46\%) with improvement in dyssynchrony.
\end{abstract}

\section{Introduction}

In recent years, His bundle pacing has emerged as a more physiologic alternative to right ventricular (RV) pacing $^{1}$. However, despite a reasonable success rate ${ }^{2}$, this procedure has yet to gain universal acceptance. Direct left bundle branch or intra-septal pacing has been recently proposed as a valid, technically feasible alternative $^{3}$ that may result in comparable benefits. Initial reports with this method have been encouraging, although long-term data remain scarce. We present a case of an adverse outcome possibly caused by direct left bundle branch pacing and reversed by an upgrade to a biventricular system.

\section{Case Report}

A 69 year old Hispanic male patient with a history of non-ischemic cardiomyopathy, normal coronary arteries by cardiac catheterization in 2011 and compensated combined congestive heart failure (EF 47\% on therapy) presented in October of 2019 with shortness of breath and lightheadedness. Initial ECG showed high grade AV block and a ventricular escape rate of $30 \mathrm{bpm}$ with a wide QRS of $120 \mathrm{~ms}$ exhibiting a right bundle branch block/left posterior fascicular block morphology (Figure 1A). His home medications included Losartan 100 $\mathrm{mg}$ a day, long-acting Metoprolol $100 \mathrm{mg}$ a day and Spironolactone $25 \mathrm{mg}$ a day.

A dual chamber pacemaker (Azure S DR MRI SureScan, Medtronic PLC, Dublin, Ireland) was implanted with insertion of a SelectSecure 3830 lead, (Medtronic PLC) intraseptally while a CapSureFix Novus 4076 lead, (Medtronic PLC) was inserted into the right atrial appendage. The 3830 lead was implanted using a stepwise approach in accordance with recently published recommendations ${ }^{4}$. The key steps included recording of a "W" shaped paced QRS complex, observation of appropriate changes in pacing impedance during advancement of the lead and ventricular activation time of $82 \mathrm{~ms}$ with both high (5.0V) and low output $(2.0 \mathrm{~V})$. The intra-septal depth of the lead tip including the screw was measured at $13 \mathrm{~mm}$ with contrast injection. 
A 12-lead ECG recorded after the implant was consistent with direct conduction system capture, possibly via the left posterior fascicle as shown in Figure 1B. The patient was discharged home on the previously mentioned medical regimen. Almost immediately he developed progressive heart failure symptoms prompting re-admission 1 month later for intravenous diuresis. Following discharge, the patient's heart failure symptoms remained refractory despite escalating doses of oral diuretics. He was re-hospitalized in February of 2020 for recalcitrant decompensated systolic heart failure. Lower extremity DVT and a small, subsegmental pulmonary embolism were concomitantly diagnosed and required the addition of anticoagulation. An echocardiogram was performed revealing a striking drop in his ejection fraction to $21 \%$ with severe wall motion abnormalities and dyssynchrony on strain imaging (Figure 2A). A right heart catheterization showed pulmonary capillary wedge pressure of $22 \mathrm{mmHg}$ and a cardiac index of $2.4 \mathrm{~L} / \mathrm{min} / \mathrm{m} 2$. The patient was treated with intravenous diuresis and afterload reduction with some symptomatic improvement but eventual worsening of his pre-renal state. He was given intravenous amiodarone to suppress frequent PVCs with improvement in this new arrhythmia burden but persistence of his symptoms. Multiple causes for worsening cardiomyopathy were considered and excluded by noninvasive and invasive testing.

The possibility of direct left bundle branch pacing as the cause for this patient's substrate and clinical decompensation was considered. In the absence of other explainable causes and given the patient's failure to respond to aggressive medical therapy a decision was made to upgrade the device to a biventricular defibrillator. A left sided pacing lead (4598, Attain Performa, Medtronic PLC) was placed into the posterolateral branch of the coronary sinus while an RV defibrillator lead (6935, Quattro Secure, Medtronic PLC) was added to the configuration and inserted at the RV apex. The direct left bundle branch lead (3830) was used as the RV pace/sense lead. The device (Amplia MRI Quad CRT-D, Medtronic PLC) was programmed to DDD mode with a lower rate limit of 60 beats per minute (bpm) with biventricular pacing at an LV to RV delay of $30 \mathrm{~ms}$. Twelve-lead ECG with pacing in this configuration is shown in Figure 1C. Paced QRS morphology is similar to direct left bundle branch pacing with some changes in terminal forces in lead I and lateral precordial leads. The chest X-ray of the final lead positioning is shown in Figure 3. A subsequent device interrogation confirmed $97 \%$ biventricular pacing.

The patient was followed after discharge and reported an immediate significant improvement in his symptoms with resolution of cough, orthopnea and improved exercise tolerance over a course of one month. An echocardiogram performed 1 month after the CRT-D upgrade showed an improvement in EF back to baseline (46\%). Strain imaging demonstrated a much more synchronous left ventricular contraction pattern (Figure $2 \mathrm{~B})$.

\section{Discussion}

This is probably the first described case of diminishing cardiac systolic function after implantation of a direct left bundle branch pacing lead reversed by the introduction of a left ventricular pacing lead. Worsening of a cardiomyopathy substrate and subsequently heart failure with His bundle pacing has not been described. This particular case highlights an incomplete understanding of the relationship between simultaneous electrical activation and cardiac synchrony. Thus, a narrower QRS complex may not always correspond to better myocardial performance. In our case a QRS of $103 \mathrm{~ms}$ was achieved with direct left bundle branch pacing. This however, by exclusion, resulted in dyssynchronous left ventricular contraction, progressive cardiomyopathy and heart failure. The timeline of heart failure events in this patient strongly suggests a poor response to direct left bundle branch pacing confirmed by resolution after an upgrade to a biventricular system. Improvement of EF after the upgrade raises the possibility of an added benefit from the lateral left ventricular wall pre-excitation in restoring cardiac synchrony in some patients. It is unclear what characteristics may define poor responders to direct left bundle branch stimulation.

\section{Conclusion}

Direct left bundle branch pacing has become a subject of interest as a novel means for ventricular stimulation and possibly cardiac resynchronization therapy ${ }^{5}$. Certain technical characteristics of this method may be complimentary to His bundle pacing. We describe the first case of worsening cardiac function and progressive 
CHF most likely attributable to direct left bundle branch pacing, both eventually corrected by the addition of the standard left ventricular pacing lead. Long term follow up series of direct left bundle branch pacing will be required to confirm the sustained benefit with this new modality.

\section{References}

1. Vijayaraman P, Chung M, Dandamudi G, Upadhyay G, Krishnan K, Crossley G, Campbell K, Lee B, Refaat M, Saksena S, Fisher J, Lakkireddy D. His Bundle Pacing. J Am Coll Cardiol. 2018 Aug, 72 (8) 927-947. DOI: 10.1016/j.jacc.2018.06.017

2. Zanon F, Ellenbogen KA, Dandamudi G, et al. Permanent His-bundle pacing: a systematic literature review and meta-analysis. Europace . 2018;20(11):1819-1826. DOI: 10.1093/europace/euy058

3. Zhang S, Zhou X, Gold M. Left Bundle Branch Pacing. J Am Coll Cardiol. 2019 Dec, 74 (24) 3039-3049. DOI: 10.1016/j.jacc.2019.10.039

4. Huang W, Chen X, Su L, Wu S, Xia X, Vijayaraman P. A beginner's guide to permanent left bundle branch pacing. Heart Rhythm 2019 Dec, 16 (12) 1791-1796. DOI: 10.1016/j.hrthm.2019.06.016

5. Wu S, Su L, Vijayaraman P, Huang Z, Whinnett Z, Huang W. Left bundle branch pacing for Cardiac Resynchronization Therapy: Non-randomized on treatment comparison with His Bundle Pacing and Biventricular Pacing. Can J Cardiol. DOI: 10.1016/j.cjca.2020.04.037

Figure 1A, B and C. Twelve-lead ECG at baseline and with different pacing configurations.

A

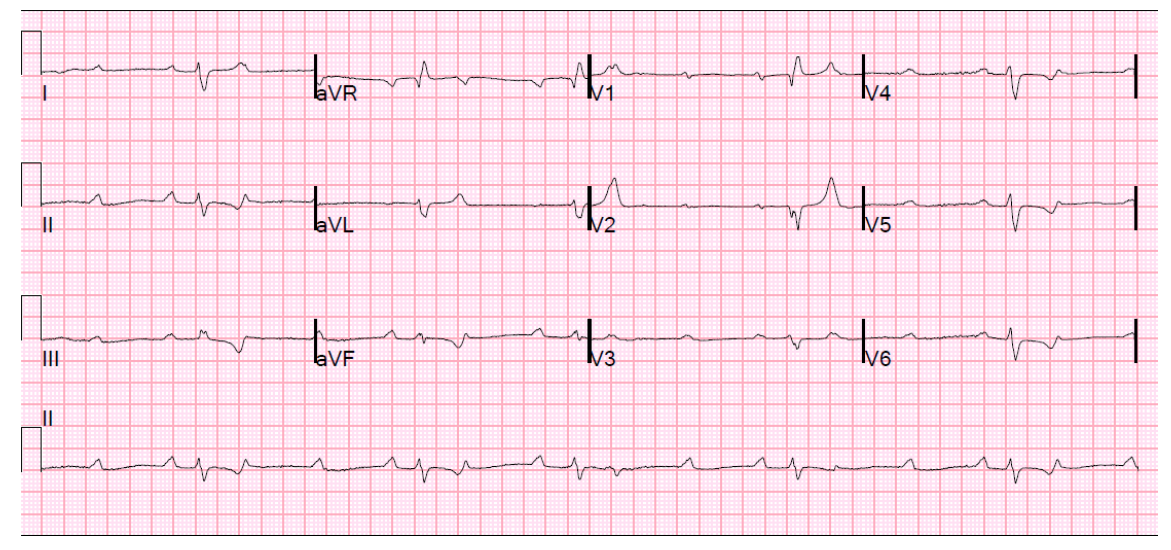

B

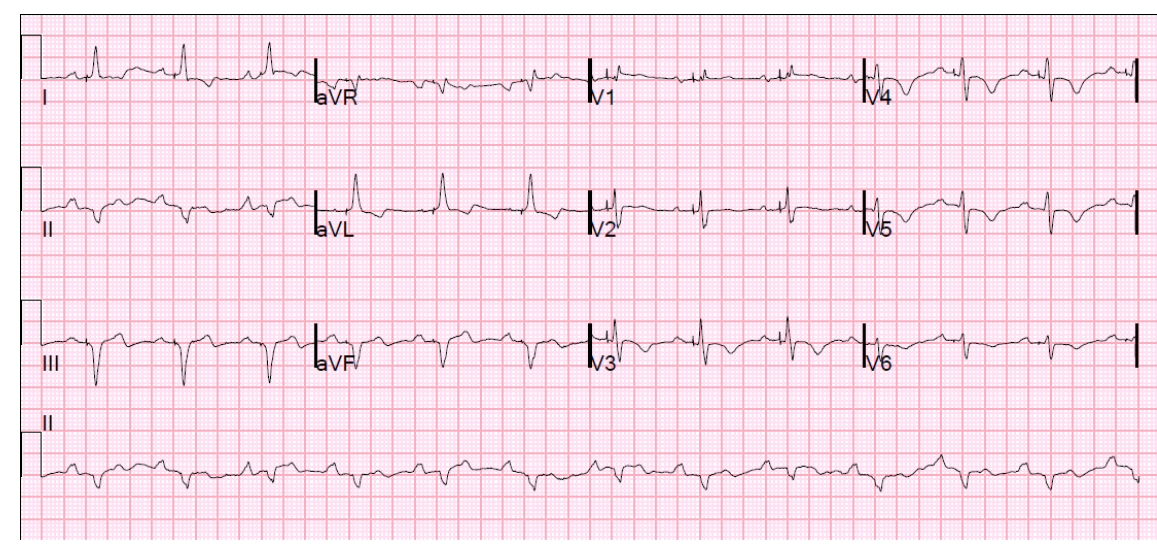

C 


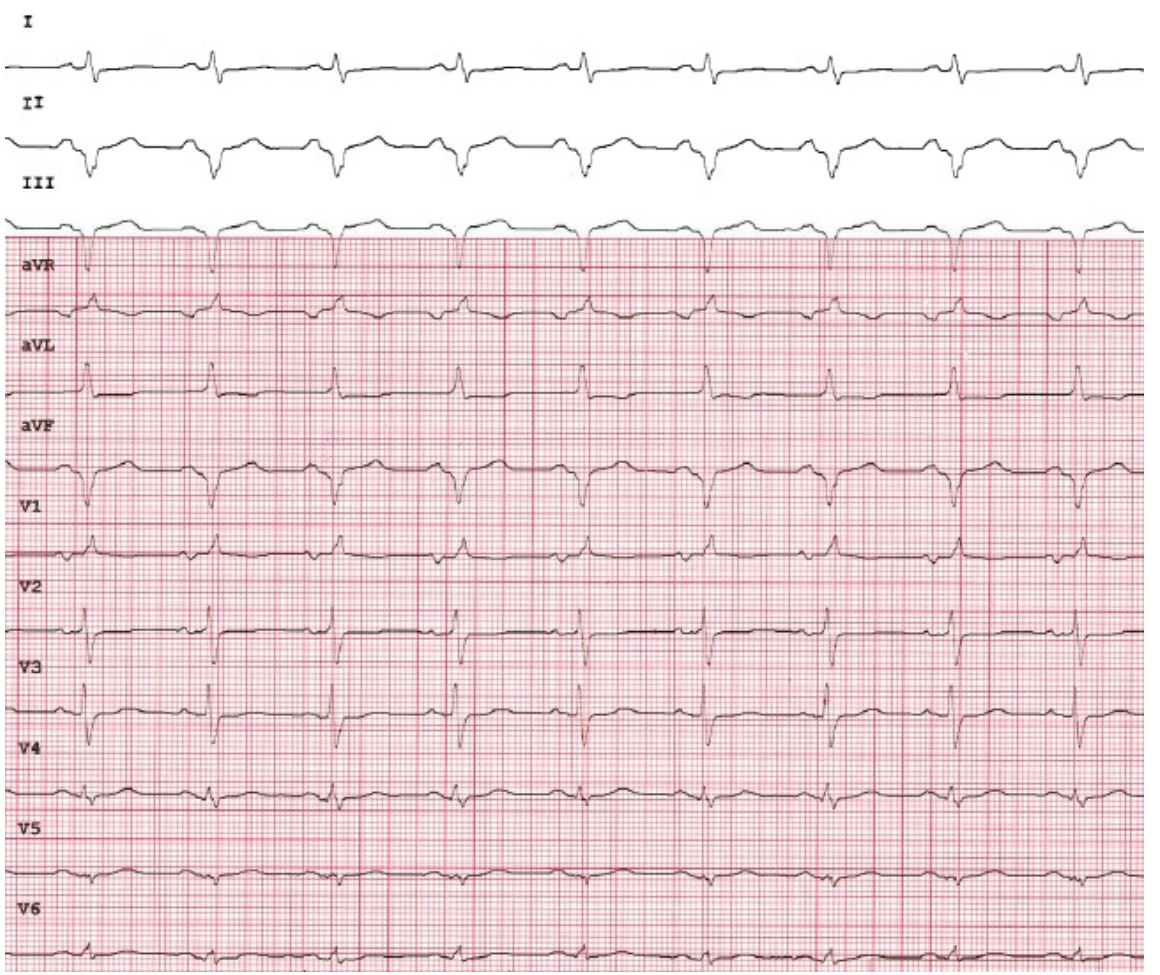

Figure legend:

A: Initial ECG upon presentation, showing high degree AV block with an escape rhythm probably arising from the left posterior fascicle. B . ECG post insertion of left bundle branch pacing lead. Notice the stimulus to QRS iso-electric segment of $30 \mathrm{~ms}$, a narrow QRS of $103 \mathrm{~ms}$ and a left anterior fascicular block pattern suggesting left posterior fascicular capture. C .ECG post CRT-D upgrade: almost simultaneous direct left bundle branch and LV postero-lateral wall stimulation, QRS morphology is similar to panel B with minor differences (see text).

Figure 2A and B. Strain imaging by echocardiogram with direct left bundle branch pacing (A) and almost simultaneous direct left bundle branch and postero-lateral LV wall stimulation (B). 

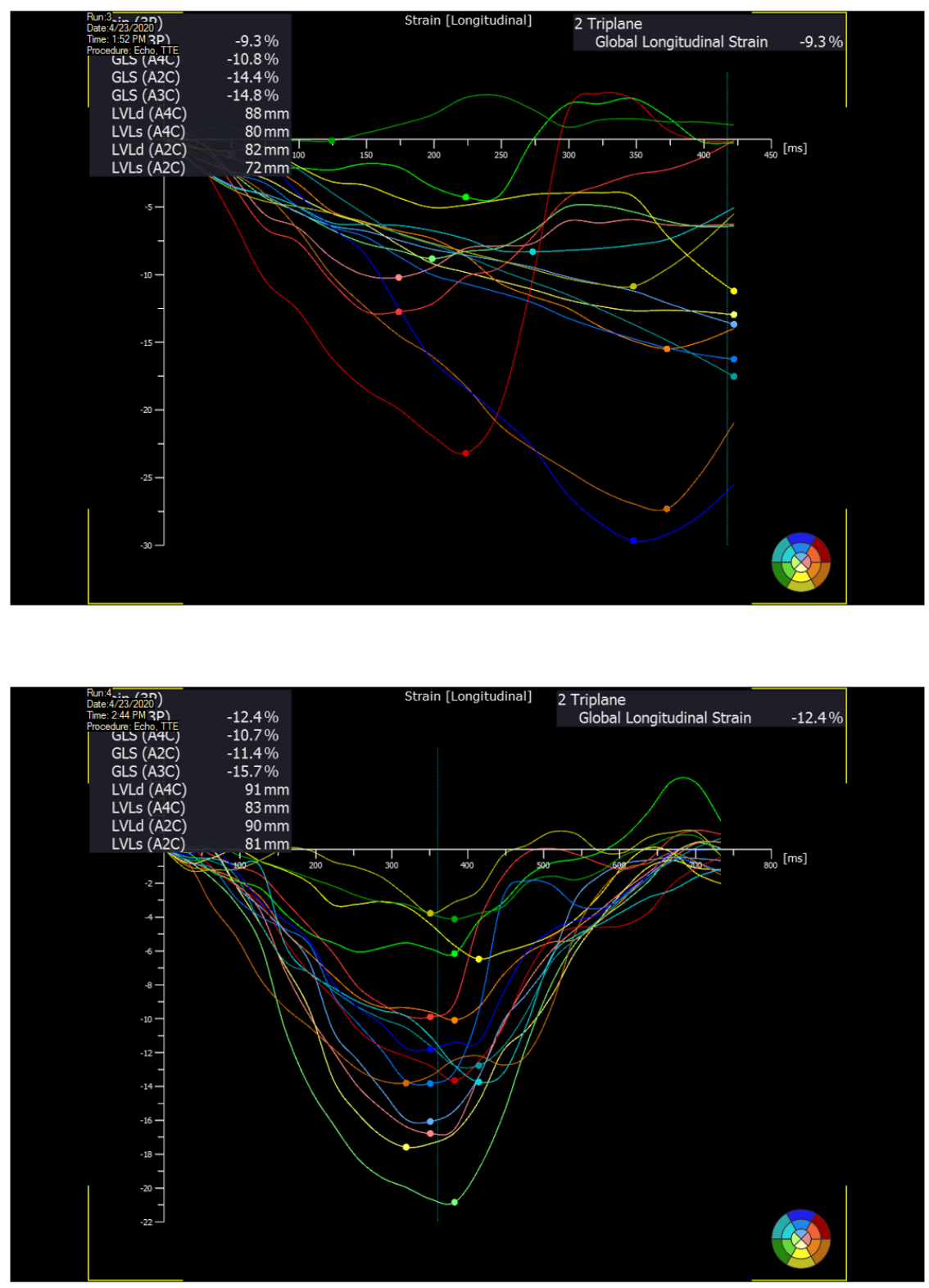

Figure legend:

In panel A, there is marked dyssynchrony among the various left ventricular wall segments indicated by different timing of the peaks as well as poor global longitudinal stain of $-9.3 \%$. In panel B a much more homogenous contraction with simultaneous peaks and an improved global longitudinal strain of $-12.4 \%$ is seen.

Figure 3. Chest X-Ray in antero-posterior view post CRT-D upgrade.

Figure legend:

Final lead positioning is shown in AP view, direct left bundle lead is deeply imbedded at the level of mid RV septum, defibrillator lead is placed at the RV apex and the CS lead is placed in a posterolateral branch of the coronary sinus. 

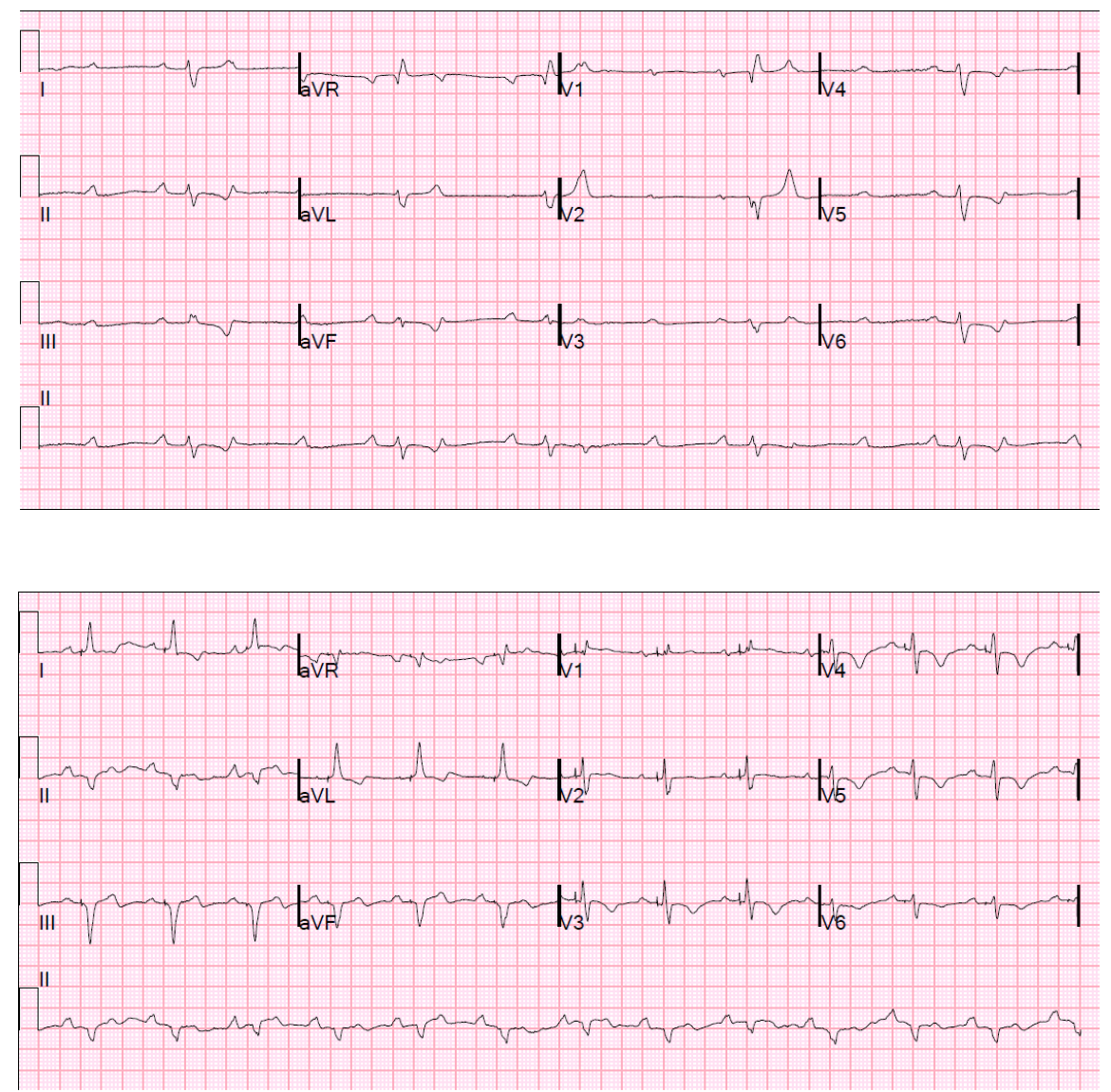

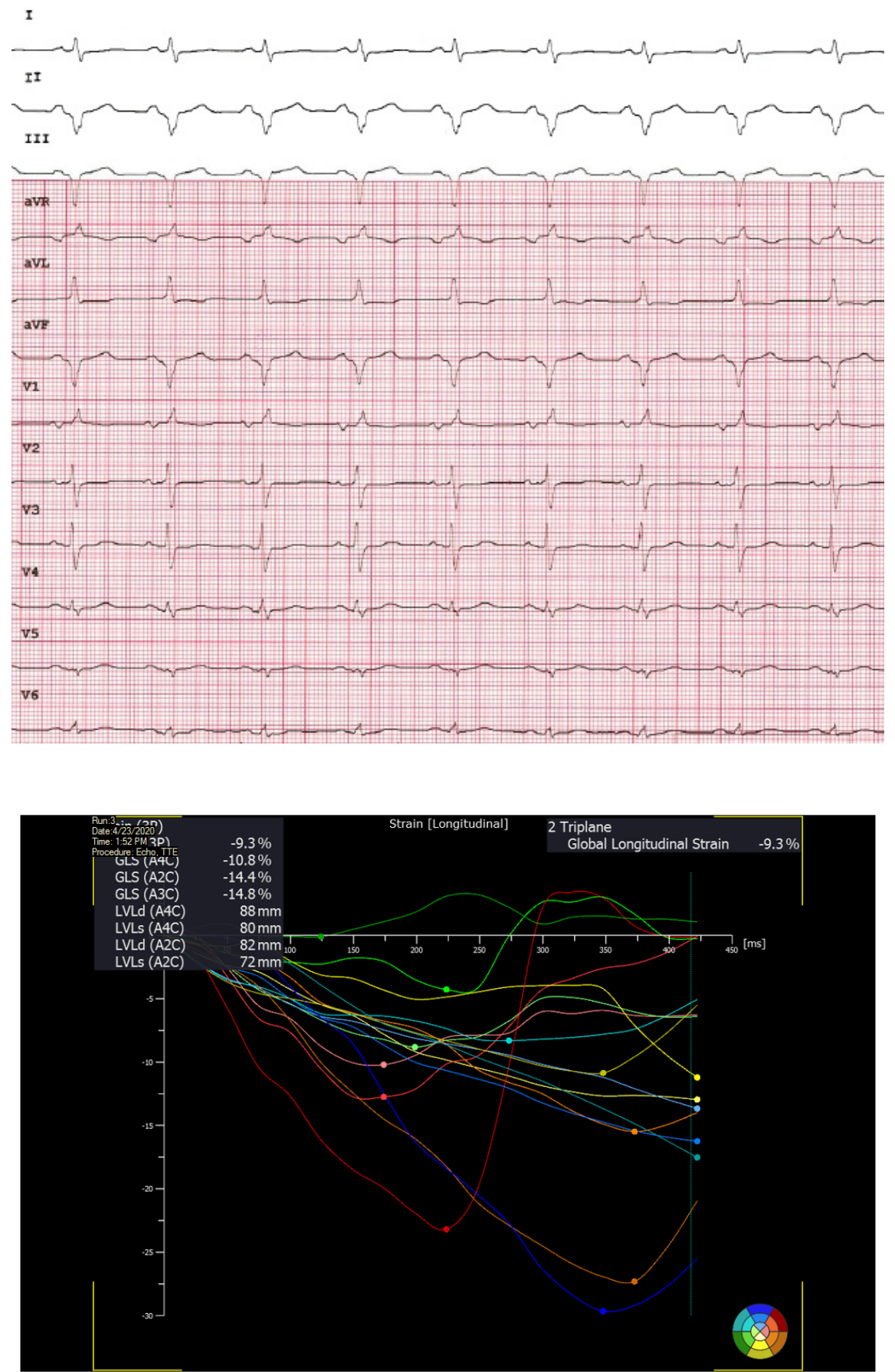

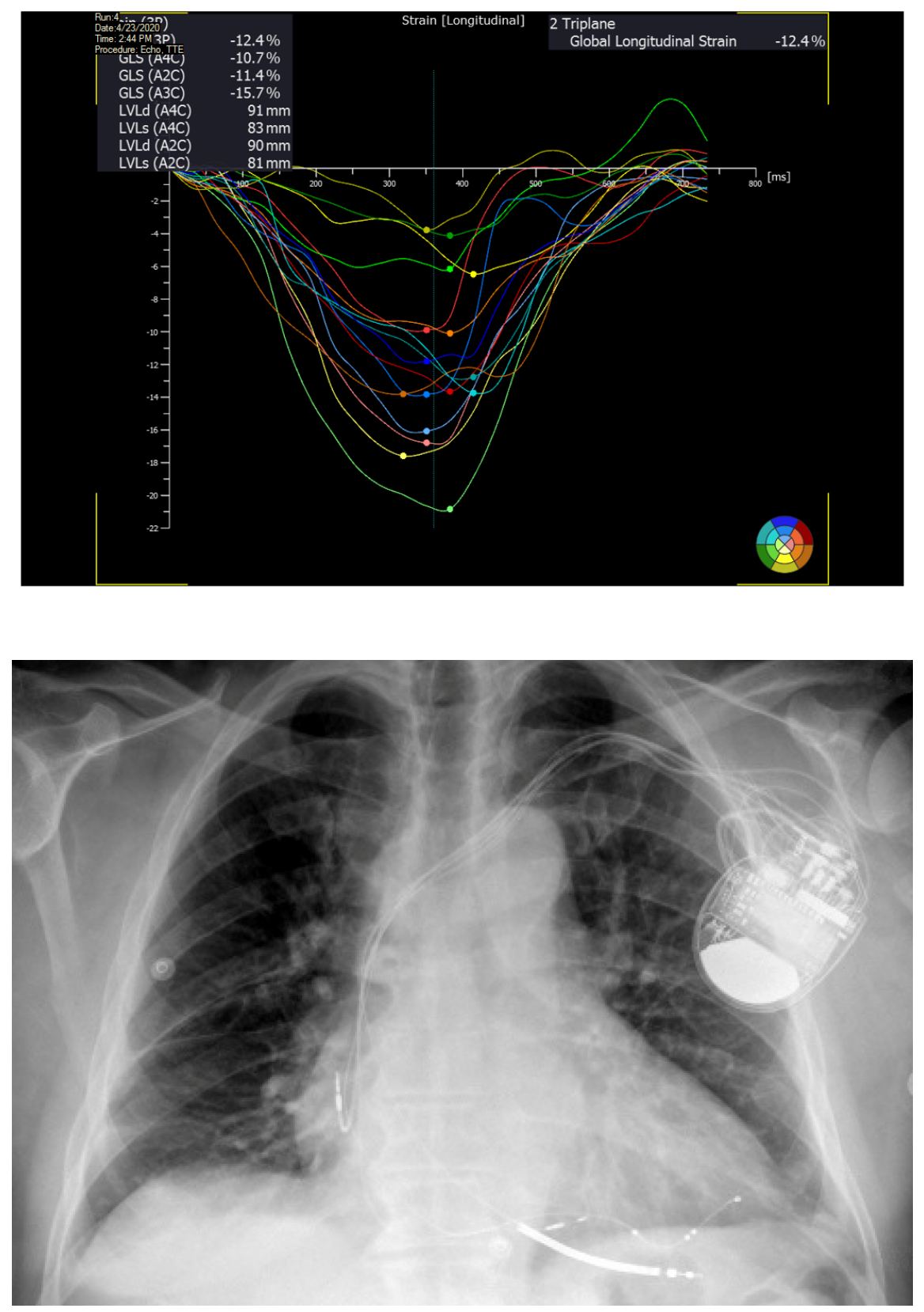